BMJ

Open

Gastroenterology

\section{The societal burden of chronic liver diseases: results from the COME study}

To cite: Scalone $\mathrm{L}$, Fagiuoli S, Ciampichini R, et al. The societal burden of chronic liver diseases: results from the COME study. BMJ Open Gastro 2015;2: e000025. doi:10.1136/ bmjgast-2014-000025

Received 2 December 2014 Revised 21 January 2015 Accepted 22 February 2015
For numbered affiliations see end of article.

Correspondence to Dr Stefano Fagiuoli; sfagiuoli@hpg23.it

\section{ABSTRACT}

Objective: Chronic liver diseases (CLDs) impose a significant socioeconomic burden on patients and the healthcare system, but to what extent remains underexplored. We estimated costs and health-relatedquality-of-life (HRQoL) among patients with CLDs at different stages and with different aetiologies.

Design: A cost-of-illness study was conducted. Direct costs, productivity loss and HRQoL were estimated in patients with chronic hepatitis, cirrhosis hepatocellular carcinoma (HCC) or where orthotopic liver transplantation (OLT) had been performed, for hepatitis $C$ virus (HCV) infection, hepatitis B virus (HBV) infection, or in those with liver disease from other causes. Patients were retrospectively observed for 6 months. The societal perspective was adopted to calculate costs.

Results: In total, 1088 valid patients (median age $=59.5$ years, $60 \%$ men) were enrolled. $61 \%$ had chronic hepatitis, 20\% cirrhosis, $8 \%$ HCC and 12\% underwent OLT. HCV infection was identified in $52 \%$ and HBV infection in $29 \%$ of the patients. Adjusted mean direct costs increased from <€200/patient-month in HCVinfected patients with hepatitis to $>€ 3000$ /patient-month in HBV infected patients with OLT. Antiviral treatment was the cost driver in patients with hepatitis, while hospital costs were the driver in the other subgroups.

Absenteeism increased from HBV-infected patients with hepatitis ( 0.7 day/patient-month) to patients with OLT with other aetiologies (3.7 days/patient-month). HRQOL was on average more compromised in cirrhosis and patients with HCC, than in hepatitis and patients with OLT. HBV-infected patients generated higher direct costs, patients with other aetiologies generated the highest productivity loss and HCV-infected patients reported the worst HRQoL levels.

Conclusions: The present study can be considered a benchmark for future research and to guide policies aimed at maximising the cost-effective of the interventions.

\section{INTRODUCTION}

Chronic liver diseases (CLDs) constitute a major medical and public health problem worldwide. According to the latest report of the European Center for Disease Prevention

\section{Summary box}

What is already known about this subject?

- Because of their relatively high prevalence and clinical consequences, chronic liver diseases (CLDs) constitute a major medical and public health problem worldwide.

- CLDs also impose a significant socioeconomic burden on affected individuals and on the healthcare system.

- In the past years, several studies have analysed implications on the burden of disease such as direct costs, or loss of productivity, or health-related-quality-of-life (HRQoL) among patients with CLDs. However, a comprehensive picture of the global socioeconomic impact of the different CLDs was still lacking.

What are the new findings?

- This study provides the most complete picture of the socioeconomic burden attributable to the different CLD stages caused by hepatitis $C$ virus (HCV) infection, hepatitis $B$ virus (HBV) infection or other aetiologies, adopting the societal perspective.

- Direct costs (ie, medical and other related costs) and loss of productivity increase with the disease progression from chronic hepatitis to cirrhosis, to hepatocellular carcinoma (HCC) and orthotopic liver transplantation (OLT), while HRQoL decreases from hepatitis to cirrhosis and HCC, but slightly improves in patients with OLT.

- HBV-infected patients generated higher direct costs, patients with other aetiologies generated the highest productivity loss and HCV-infected patients reported the worst HRQoL levels.

and Control, ${ }^{1}$ the prevalence of chronic hepatitis $B$ virus (HBV) infection in the general population ranges from $0.2 \%$ to over $7 \%$ in the different European countries, while the prevalence of hepatitis $\mathrm{C}$ virus (HCV) varies from $0.4 \%$ to over $3 \%$ in Mediterranean countries. However, as high-risk and vulnerable groups such as migrants, homeless persons and prisoners tend to be under-represented in general population studies, the HCV and HBV prevalence data are likely underestimated. 


\section{Summary box}

How might it impact on clinical practice in the

foreseeable future?

- The use of effective interventions such us screening and treatments, implemented from the early stages of liver diseases, appears necessary to reduce worsening of patient health, and direct and indirect costs. The availability of new antiviral therapies is expected to change the medical approach towards patients with CLDs as well as the treatment. A proper knowledge of the global socioeconomic burden of CLDs is paramount to plan adequate screening and treatment policies aimed at maximisation of cost-effectiveness of interventions. The present study can be considered a benchmark for future research and to guide policies aimed at maximising the costeffective of the interventions.

Individuals with chronic hepatitis B and/or $\mathrm{C}$ virus infection remain infectious to others and are at risk of developing serious liver diseases such as cirrhosis and hepatocellular cancer (HCC) later in life. ${ }^{2-4}$ The natural history and progression of chronic HBV-related and HCV-related diseases is complex, highly variable and can involve individuals for most of their life ${ }^{5}{ }^{6}$ However, many patients who might benefit from treatment still remain undetected, allowing progression of the disease with severe consequences in terms of healthcare resource consumption and quality of life. ${ }^{1}$ Participants at a recent hepatitis $\mathrm{B}$ and $\mathrm{C}$ summit conference ${ }^{7}$ underlined that although the threat posed by chronic viral hepatitis is becoming more apparent in Europe, there is still little understanding at the public or policy level of the health and implications of hepatitis B and C, from a healthcare as well as societal point of view. CLDs also impose a significant socioeconomic burden on affected individuals and on the healthcare system. In recent years, several studies have been conducted to quantify some implications regarding the burden of the diseases, such as direct costs, or loss of productivity or health-related-quality-of-life (HRQoL) among patients with CLDs. However, most of the studies were conducted in the Americas or in Asiatic countries, or focused on HCV infection or sequelae..$^{8-19}$ A comprehensive picture of the global socioeconomic impact of the different CLDs is still lacking. Knowledge of the global socioeconomic burden of this complex phenomenon is paramount for planning adequate screening and treatment policies aimed at maximising the benefits and efficiency of treatment strategies.

The aim of the present study was to estimate the socioeconomic impact and HRQoL among patients diagnosed with CLDs at different stages and with different aetiologies.

\section{PATIENTS AND METHODS}

Study design

A retrospective, prevalence-based cost-of-illness (COI) study, named COME (COsti delle Malattie Epatiche), involving the enrolment of patients over 1 year, was conducted.
In our study, we estimated medical and non-medical costs (direct costs), loss of productivity (indirect costs) and health-related quality of life (intangible costs) in patients with a confirmed diagnosis of CLDs. To conduct this study, we adopted the society's point of view, that is, the point of view of patients and their family/caregivers (relatives or friends), and of the third party payer, which in Italy is the National Health Service. To collect economic data, we adopted a retrospective time horizon of 6 months before enrolment, which in previous similar studies on patients affected by chronic diseases such as CLDs has been shown to be a reasonable time period. ${ }^{20}$

\section{Participants and setting}

We sequentially enrolled adult patients with CLDs, for around 1 year, at the Gastroenterology Unit of two tertiary centres in Italy: Papa Giovanni XXIII Hospital of Bergamo and Federico II University hospital in Naples. The recruitment time of 1 year was decided as recognised suitable to be able to recruit all the target patients who were in care at the participant centres during the study conduction. To be eligible, patients had to meet the following criteria: age $>18$ years, diagnosis of CLD, and ability to understand the objective and contents of the study, and to report the information required. Eligible patients had to sign an informed consent form, after receiving information, on the aim of the study, the type of data and the method of data collection. The participants were classified according to the most advanced condition diagnosed (ie, chronic hepatitis, cirrhosis, hepatocellular carcinoma) or whether an orthotopic liver transplantation (OLT) had been performed or not. Patients classified as with chronic hepatitis were by definition without cirrhosis or hepatocelluar carcinoma, and were patients on active antiviral treatment, or on posttreatment follow-up (both responder or not), or on regular surveillance follow-up for CLD. As regards patients with cirrhosis, the diagnosis of this condition was either histological or clinical, the latter based on imaging (US scan, CT scan), biochemical (ie, low platelets level) and/or according to clinical evidence of cirrhosis. The patients were also classified into three groups according to the earliest aetiological factor identified: HCV infection, HBV infection or other causes, which included viral coinfections, alcoholic liver disease, cholestatic disease and autoimmune disease. The study was conducted in agreement with the National Regulatory Requirements, International Conference on Harmonization Guidelines for Good Clinical Practice and the 18th World Medical Assembly, ${ }^{21}$ and all subsequent amendments. The study protocol was accepted by the ethics committees of the two participating hospitals.

\section{Data collection and costs estimate}

The following data were collected at the enrolment of each patient: sociodemographics and information on 
habits, on diagnosis and aetiology, on resource consumption for reasons attributable to the liver disease and on HRQoL. Depending on the type of data, these were reported by the physicians (eg, data on diagnosis and aetiology) or by the patients themselves (eg, HRQoL, loss of productivity) or by the physicians after asking the patients about details (eg, on medical visits or drug treatment). Ad hoc structured questionnaires were prepared and used to collect the data: the patients were asked to autonomously complete a paper questionnaire containing the standard version of the HRQoL questionnaire (see later for details), questions on loss of productivity and on non-conventional treatment (ie, homeopathic products, herbal drugs, vitamins, antioxidant or supplement products, alternative techniques/therapies, special diets) used for reasons attributable to their liver condition. The physicians completed an electronic database in which they input information gathered from medical records (eg, clinical data) or specified by the patients (eg, treatment details).

As regards resource consumption, to estimate direct medical costs we collected information on conventional drug treatment and on non-conventional treatment to treat the liver disease, on hospitalisations, and on outpatient specialist medical visits and diagnostic tests attributable to the liver disease. Data on the amount of non-medical resource consumption attributable to the liver disease (ie, on travel and/or accommodation to reach the hospital or medical practice, and on formal caregiving, such as paid assistance at home) were also collected.

To convert these data to monetary terms, we multiplied the amount of resources consumed by their unit cost derived from tariffs or prices applicable in Italy in 2011. In particular, medical costs were quantified using prices/tariffs paid by the National Health Service: the Italian Drug Agency price list ${ }^{22}$ was used for drug costs. Costs of hospital admissions were calculated according to the diagnosisrelated group regional tariffs. ${ }^{23}$ Costs for outpatient services were obtained from the regional outpatient lists. ${ }^{24}$

The medical services paid by the patients (provided as private sector care) were quantified utilising the information on the out-of-pocket cost specified by each participant.

Also, we collected information on productivity lost by the patients themselves or by their informal caregivers (ie, family members or friends) who mainly helped the patients to manage their condition. The patients were asked to specify whether, during the previous 6 months, they had had any quality/efficiency reduction in working, studying or performing any other everyday activity. They were also asked to report the number of lost days/hours of productivity, if any, and to specify whether they had changed or lost their job for reasons attributable to their hepatic condition in the previous 6 months. Furthermore, we collected data on the number of days or hours of work the informal caregiver lost to help the patient with his/her condition.
HRQoL was assessed using the EQ-5D questionnaire, which is a generic HRQoL instrument for describing and valuing health, ${ }^{25}$ very useful to calculate utility for the obtainment of quality-adjusted life years (QALYs), which is a useful outcome indicator for pharmacoeconomic evaluations (cost-utility analyses) of treatment options. With the EQ-5D, the respondents are asked about their HRQoL on the current day. The tool consists of two parts: a descriptive system (EQ-5D profile) consisting of five domains, namely 'mobility', 'self-care', 'anxiety/depression', 'usual activities' and 'pain/discomfort'. The standard version of the descriptive system includes three levels of severity per domain ('no problem', 'some/moderate problems', 'extreme problems/impossible to do').

We also used a new version of the EQ-5D descriptive system, with five levels of severity for each domain (EQ-5D-5L): 'no problem', 'mild problems', 'moderate problems', 'severe problems' and 'extreme problems', with the aim of obtaining more precise data from patients with CLDs. We compared the performance of the EQ-5D-5L with the standard 3L version in this category of participants. Results on this comparison have been published elsewhere. ${ }^{26}$ The second part of the questionnaire consists of a visual analogue scale (EQ-5D VAS) measuring the overall HRQoL, ranging from 0 (worst imaginable health state) to 100 (best imaginable health state).

In this paper, we report the HRQoL results obtained with the VAS and as utility indexes. To calculate the utility index from the data obtained with the EQ-5D-3L descriptive system (named 3L utility index), the Italian-specific social tariffs were used, ${ }^{27}$ while the data collected with the EQ-5D-5L descriptive system were converted into utilities (named 5L utility index) using the mapping algorithm developed by van Hout $e t a l^{28}$ and applied on the Italian social tariffs.

\section{Data analyses}

Sociodemographic and clinical characteristics of the study sample, and resource consumption for all categories, are described with proportions for categorical data, mean as the central tendency parameter for continuous data, and minimum and maximum values as dispersion parameters.

In particular, consumption of resources related with direct costs is expressed as the frequency of patients consuming each item, or the frequency of cost items (eg, hospital accesses, outpatient claims) occurring in the different subgroups of patients. Costs for each category were then quantified in monetary terms and expressed as mean (minimum-maximum) $€$ /patient-month.

Loss of productivity results are reported as the proportion of patients with reduced efficiency and/or who lost days or hours of work/study/everyday activities. These are also expressed in terms of mean (minimummaximum) days/patient-month of productivity loss. 
HRQoL is reported as mean of the VAS score and mean of the utility indexes estimated from the EQ-5D-3L, and from the EQ-5D-5L.

Direct costs, loss of productivity and HRQoL can be influenced by the patients' characteristics or events; while some characteristics or events are directly related with the target condition or its management, for example, disease stage, aetiology, treatment, surgery, other aspects such as age, gender, education, working status, etc, can be related with costs and/or HRQoL regardless of liver condition, as 'confounders'. We aimed to estimate costs and HRQoL in each stage of the CLDs and according to each aetiology, adjusting the estimates for the other aspects that could be related with costs or HRQoL as confounders. Accordingly, we conducted multivariable regression models in which the dependent variables, that is, the variables that we aimed to estimate, were total direct costs, loss of days of productivity or VAS or utility index scores for HRQoL. As independent (explanatory) variables, the models included the disease condition and aetiology in order to obtain estimates specific for each subcategory of patients, plus those variables that were considered potential confounders and for which we aimed to adjust for. The choice of these confounding variables was made according to knowledge from past experience in the areas of CLDs and of health economics, from the literature that was available at the time of this study conduction, and according to the performance of the statistical models.

In particular, we adopted the general linear models (GLM) to estimate direct and indirect mean costs, because these variables present a highly skewed distribution (instead of a Gaussian distribution), that is, few participants generate very high costs, while most generate low costs. Furthermore, costs cannot be of a negative sign, hence the lowest possible value corresponds to 0 , and can theoretically be of an infinite amount. Family and link functions of the GLM model were chosen by the Modified Park Test for the former and by the Pearson Correlation and the Pregibon Link tests for the latter, resulting in a model with $\gamma$ family and root square link functions. With these models, direct costs and loss of days of productivity (dependent variables) were estimated as mean $€ /$ patient-month and mean days lost from everyday activities/patient-month, respectively. The independent variables included in these models were: disease stage and aetiology to obtain estimates in each specified subgroup, while age, gender, working status, marital status, time from diagnosis, occurrence of examinations for evaluation of liver transplantation, and current and type of therapy were added as possible confounders.

To estimate the mean VAS score and utility indexes, we applied the linear regression models using the Ordinary Least Square method. The following independent variables were included: disease condition and aetiology to obtain estimates in each subgroup specified, while age, gender, education, working status, marital status, alcohol use, time from diagnosis, and current and type of therapy were added to adjust for these possible confounders.

Regression coefficient estimates, z (GLM), t and $p$ values are reported for each model.

As regards the management of missing data: if missing data from one item were few and did not interfere with the analyses, they were ignored, otherwise, they were filled using means or most frequent value calculated from the available data of the same variable. If missing data of an item were many, the related variable was not included in the analyses. If missing data from one participant were many, all the data from that participant were not included in the analyses.

All analyses were performed using SPSS V.15.0 software (SPSS, Chicago, Illinois, USA) and with STATA V.11 version (StataCorp LP, USA).

\section{RESULTS}

Sample description

From January 2010 to December 2011, data from 1091 consecutive patients were collected. The data of three patients were excluded from the analyses because two patients withdrew their informed consent after completing the questionnaire, and too few data were available from one patient. Hence, the study sample involved 1088 patients aged $18-89$ years, $60 \%$ men. Description of the study sample is reported in table 1 . As regards patients belonging to the OLT subgroup, $12(9.3 \%$ of the transplanted patients) underwent OLT within the 6 months prior to enrolment.

\section{Medical resources consumption \\ Treatment}

During the observational period, $35.6 \%$ of the patients did not receive any treatment for their hepatic condition, $34.7 \%$ received only conventional drug treatment, $18.9 \%$ underwent conventional and unconventional treatments, and $10.7 \%$ underwent unconventional treatment alone. In particular, antiviral drug treatment was taken by $24 \%$ of the full study sample. Table 2 shows the proportion of patients taking antiviral treatment stratified according to their condition and aetiology.

Patients reporting having received unconventional treatment during the observational period $(35.8 \%$ in total) more frequently took vitamins, antioxidant or supplement products $(59.4 \%$ of patients using unconventional treatment) and special dietary products $(37.5 \%)$, while up to $33.7 \%$ took homoeopathy and/or herbal medicines and/or used alternative techniques/ therapies.

\section{Hospitalisations and outpatient accesses}

Overall, $34.7 \%$ of patients were hospitalised one or more times as day-hospital and/or ordinary regimen admission during the observational period: $19.6 \%$ of the patients who were hospitalised had hepatitis $(81 \%$ of 


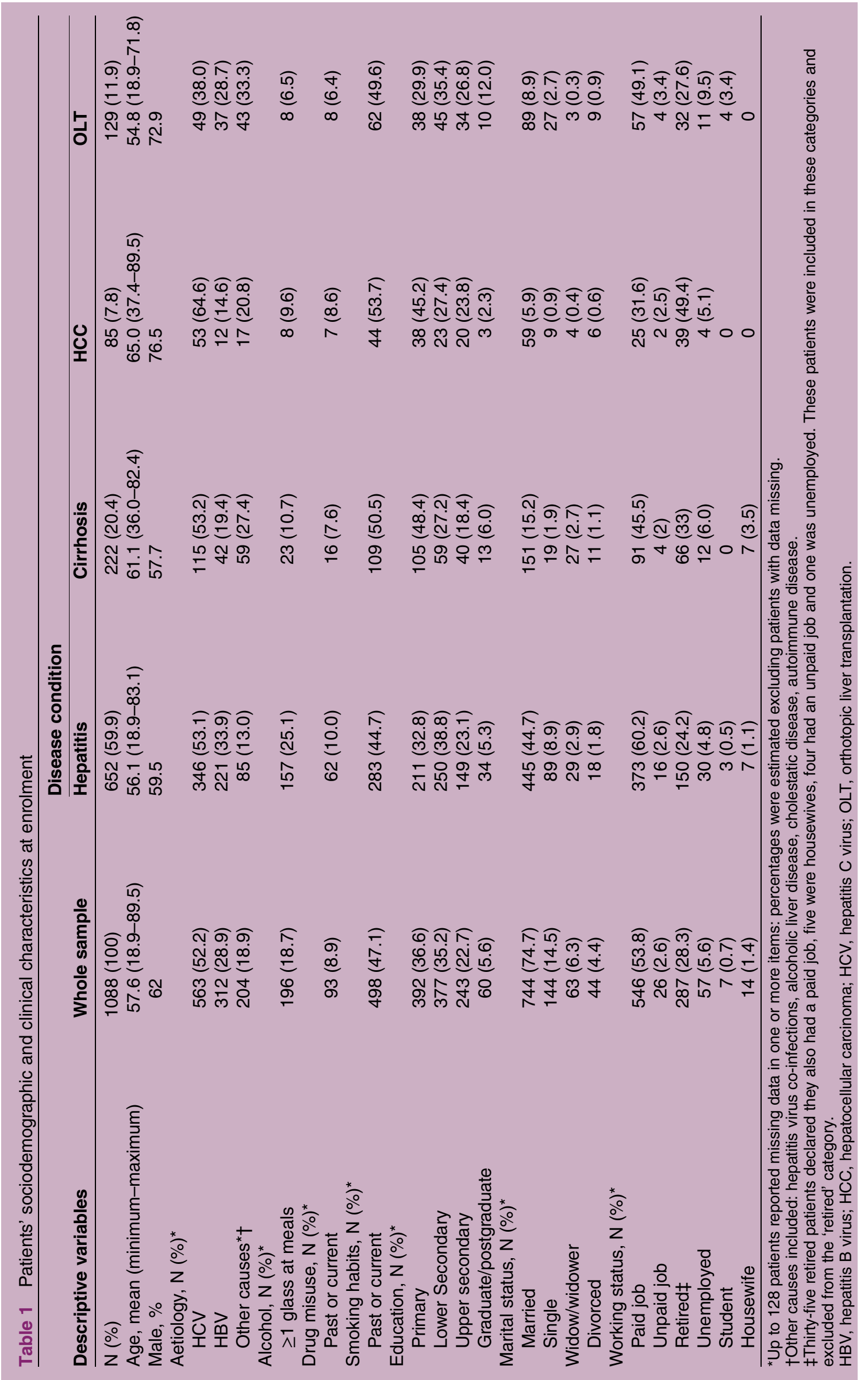


Table 2 Patients who took antiviral treatment, stratified by aetiology and disease condition

\begin{tabular}{|c|c|c|c|c|c|}
\hline Aetiology & $\begin{array}{l}\text { Hepatitis } \\
\mathrm{N}\left(\%^{\star}\right)\end{array}$ & $\begin{array}{l}\text { Cirrhosis } \\
N\left(\%^{\star}\right)\end{array}$ & $\begin{array}{l}\text { HCC } \\
N(\% *)\end{array}$ & $\begin{array}{l}\text { OLT } \\
\text { N (\%*) }\end{array}$ & $\begin{array}{l}\text { Total by aetiology } \\
\text { N (\%*) }\end{array}$ \\
\hline $\mathrm{HCV}$ & $42(12.1)$ & $3(2.6)$ & $1(1.9)$ & $16(2.8)$ & $62(11.0)$ \\
\hline HBV & $111(50.2)$ & $21(50.0)$ & $10(83.3)$ & $37(100)$ & $177(56.7)$ \\
\hline OTHER causes $†$ & $3(3.5)$ & $3(5.1)$ & $3(17.6)$ & $11(25.6)$ & $20(9.8)$ \\
\hline Total by disease condition & $156(23.9)$ & $27(12.5)$ & $14(17.1)$ & $64(49.6)$ & $259(24.0)$ \\
\hline
\end{tabular}

those admitted as day-hospital regimen, $21 \%$ admitted in ordinary regimen), $28.3 \%$ had cirrhosis $(73.1 \%$ of those admitted as day-hospital, $45 \%$ admitted in ordinary regimen), $18 \%$ had HCC $(77.1 \%$ of those admitted as day-hospital, $45.7 \%$ admitted in ordinary regimen) and $34.1 \%$ had undergone OLT $(100 \%$ of those admitted as day-hospital and $34 \%$ admitted also in ordinary regimen).

The overall number of hospitalisations during the observational period was around 1400, with $86 \%$ occurring as day-hospital regimen. Overall, an average of 1.4 accesses per patient-month occurred: 1.3 among patients with hepatitis, 1.5 among those with cirrhosis, 1.6 among patients with HCC and 2.6 accesses per patient-month among patients with OLT. The most frequent hospital accesses occurred for post-OLT follow-up (36.5\% of all hospital accesses) or OLT evaluation (25.8\%).The patients were mainly hospitalised for diagnostic reasons, representing $94 \%$ of hospital accesses in hepatitis and transplanted patients, and $82-83 \%$ in those with cirrhosis or HCC. The other hospital accesses occurred for therapeutic reasons.

Outpatient accesses occurred in $85.6 \%$ of the study sample. In particular, $20.8 \%$ of patients with at least one outpatient access had hepatitis, 28.2\% had cirrhosis, $18.2 \%$ had HCC and $32.7 \%$ were patients with OLT. Around 4200 outpatient accesses occurred in total, corresponding to a mean of 0.6 accesses per patient-month, $98 \%$ of which were due to specialist medical visits or laboratory/instrumental tests.

\section{Direct costs}

From the crude estimates we found that the mean total direct costs increase from hepatitis to the more advanced conditions (table 3 ). However, some cost items contributed differently to the total direct costs between the different disease conditions: while the cost of conventional treatment was on average the driver among patients with hepatitis, followed by hospitalisations, this cost item generated the highest mean costs, and conventional drug treatments generated the second most important cost item in patients with cirrhosis, HCC and OLT. In the OLT subgroup, which generated the highest hospital mean costs, $40.2 \%$ of the inpatient costs were attributable to the 12 patients who underwent liver transplantation within the 6 months before enrolment.

As regards conventional treatment, in patients with hepatitis, costs were $97.6 \%$ attributable to antiviral treatment, whereas in HCC and patients with OLT, antiviral treatment contributed to $45 \%$ and $14 \%$ of treatment costs, respectively.

If we focus on patients who received antiviral treatment, those with $\mathrm{HBV}$ infection cost $€ 471.9 /$ patientmonth in patients with hepatitis, $€ 380.9 /$ patient-month in the cirrhosis subgroup, $€ 374.9 /$ patient-month in the HCC subgroup and $€ 176.3 /$ patient-month in the OLT subgroup.

Meanwhile, among patients with $\mathrm{HCV}$ infection receiving antiviral treatment, it cost $€ 1269.2 /$ patient-month in those with hepatitis, $€ 463.8 /$ patient-month in cirrhotic patients, €433.2/month in the only HCC patient receiving antiviral treatment and $€ 768.4$ /patient-month in the patients with OLT.

As regards the other direct costs, non-medical costs were the third (cirrhosis, HCC and OLT) or fourth most important cost item, while unconventional treatment (hepatitis, cirrhosis and HCC) and outpatient (OLT) costs were the least expensive items.

From the regression model (table 4 ) we found a statistically significant $(\mathrm{p}<0.01)$ increase of the mean total direct costs in cirrhosis, HCC and patients with OLT compared with patients with hepatitis. In contrast, the differences between the aetiological subgroups were not statistically significant $(p>0.1)$. Other variables that were found to be significantly related with total direct costs were the occurrence of evaluation for liver transplantation $(\mathrm{p}<0.001)$, and if the patients were following a drug therapy $(p<0.01)$. Figure $1 \mathrm{~A}$ shows the trends of the mean total costs estimated in each group of patients defined for diagnosis and aetiology, adjusted for the possible confounders included in the regression model.

\section{Loss of productivity}

Almost half $(44.7 \%)$ of the participants reported that they had less efficiency and/or more lost time in doing their everyday activities during the previous 6 months. Twenty patients also specified that they lost their job for reasons attributable to their condition: seven patients with hepatitis, six with cirrhosis, one with HCC and six 


\begin{tabular}{|c|c|c|c|c|}
\hline \multirow[b]{2}{*}{ Cost category } & \multicolumn{4}{|l|}{ Disease condition } \\
\hline & $\begin{array}{l}\text { Hepatitis } \\
\text { Mean (minimum- } \\
\text { maximum) }\end{array}$ & $\begin{array}{l}\text { Cirrhosis } \\
\text { Mean (minimum- } \\
\text { maximum) }\end{array}$ & $\begin{array}{l}\text { HCC } \\
\text { Mean (minimum- } \\
\text { maximum) }\end{array}$ & $\begin{array}{l}\text { OLT } \\
\text { Mean (minimum- } \\
\text { maximum) }\end{array}$ \\
\hline \multicolumn{5}{|l|}{ Medical costs } \\
\hline $\begin{array}{l}\text { Conventional, antiviral drug } \\
\text { treatment }\end{array}$ & $167.1(0-1921.9)$ & $47.9(0-597.2)$ & $62.8(0-508.8)$ & $150.6(0-1830.0)$ \\
\hline $\begin{array}{l}\text { Conventional, other } \\
\text { (non-antiviral) drug } \\
\text { treatment }\end{array}$ & $4.0(0-325.5)$ & $99.1(0-1543.5)$ & $75.1(0-1038.6)$ & $917.9(25.2-6052.0)$ \\
\hline Unconventional treatment & $10.2(0-533.3)$ & $22.5(0-400.0)$ & $17.3(0-207.7)$ & $16.8(0-300.0)$ \\
\hline Hospitalisations & $49.0(0-3075.0)$ & $259.3(0-4612.5)$ & $999.8(0-2590.3)$ & $1483.9(38.0-13370.3)$ \\
\hline $\begin{array}{l}\text { Outpatient visits/ } \\
\text { examinations }\end{array}$ & $16.9(0-163.2)$ & $25.5(0-105.0)$ & $23.6(0-141.3)$ & $8.2(0-38.7)$ \\
\hline \multicolumn{5}{|l|}{ Non-medical costs } \\
\hline Formal caregiving & $2.7(0-500.0)$ & $9.2(0-500.0)$ & $9.3(0-166.7)$ & $17.8(0-300.0)$ \\
\hline $\begin{array}{l}\text { Travelling and } \\
\text { accommodation }\end{array}$ & $12.8(0-500.0)$ & $38.3(0-500.0)$ & $41.2(0-375.0)$ & $79.5(0-1166.7)$ \\
\hline Total direct costs & $262.8(3.7-3166.8)$ & $501.7(7.9-4693.6)$ & $1229.2(11.4-3916.0)$ & $2674.7(155.7-15669.7)$ \\
\hline
\end{tabular}

who underwent OLT. As regards the time lost from everyday activities, an average of 0.7 days/patient-month were lost by the patients overall. Moreover, $44.8 \%$ patients declared they received help from informal caregivers, with an estimate of a further 0.3 days/patientmonth of caregivers' productivity lost, across the full study sample.

From the regression model (figure 1B) we found an increase of the mean loss of productivity in cirrhosis (not significant, $\mathrm{p}>0.200$ ), HCC and in patients with OLT $(\mathrm{p}<0.05)$ compared with patients with hepatitis. In contrast, the differences between the different aetiological subgroups were not statistically significant ( $p>0.1)$. Other variables that were found to be significantly related with the loss of productivity were the occurrence of drug therapy $(\mathrm{p}<0.05)$ and HRQoL measured with the VAS. Figure 1A shows the trends of the adjusted mean loss of productivity estimated in each group of patients defined for diagnosis and aetiology.

\section{Health-related quality of life}

The model results for the VAS score, the $3 \mathrm{~L}$ and the $5 \mathrm{~L}$ utility indexes, are detailed in table 4 . Considering the other variables included in the model equal, patients with cirrhosis had lower levels than patients with hepatitis, those with HCC had lower levels than patients with cirrhosis, while in patients with OLT, HRQoL levels almost reached, on average, the same levels as those of patients with hepatitis. However, these differences were generally not statistically significant. As regards aetiology, HCV patients had lower levels than HBV patients and patients with other causes, making these differences generally statistically significant. Figures 1C, 1D and 1E show the estimates for each category of interest and have been adjusted for all potential confounding variables included in the models. As regards the VAS (figure 1C), the adjusted mean values decrease from hepatitis to cirrhosis and HCC, finally they increase again in patients with OLT, almost reaching the scores estimated for patients with hepatitis. Patients with HCV reported the worst mean levels of HRQoL, followed by patients with other aetiologies and then by patients with HBV infection. This trend is somewhat similar to those found for the $3 \mathrm{~L}$ (figure 1D) and the 5L (figure 1E) utility scores.

\section{DISCUSSION}

To the best of our knowledge, this study provides the most complete picture of the socioeconomic burden attributable to the different CLDs caused by HCV infection, HBV infection or other aetiologies, from the societal perspective, that is, considering the point of view of the third party payers, the patients and the patients' family/caregivers.

Direct costs had an increasing trend from patients with hepatitis (less than $€ 400 /$ patient-month) to those with cirrhosis (up to $€ 700 /$ patient-month), HCC (up to $€ 2600$ /patient-month) and OLT (up to €3000/patientmonth), with some differences detected within the three aetiological subgroups: within the same disease conditions, patients with HBV infection generated on average the highest direct costs, followed by patients with other aetiologies and then by those with HCV infection. In particular, antiviral treatment was the cost driver in patients with hepatitis, while hospitalisations generated the highest mean costs in the other conditions. As regards patients receiving antiviral drug treatment during the observational period, patients with hepatitis generated the highest mean costs, corresponding to $€ 500 /$ patientmonth among hepatitis $\mathrm{B}$ patients taking antivirals, and $€ 1300$ /patient-month among hepatitis $\mathrm{C}$ patients. 
ชิ ชิ สิ

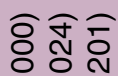

क्ष $\widehat{్}$

ơ

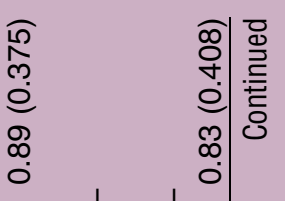

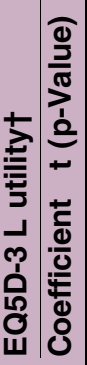
○ लํ

iอ

อง

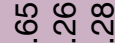

Mํ.

ल กั।

อ

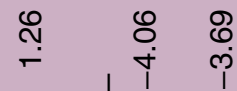

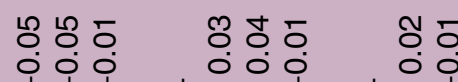

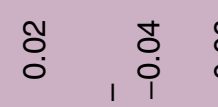

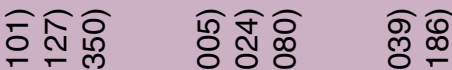

¿ंe

సิ จิ $\overline{8}$

क्

- 0 0

กุ?

$\begin{array}{lll}\delta & & \vdots \\ 0 & & \\ 0 & & 0\end{array}$

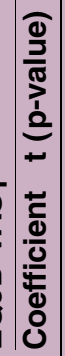

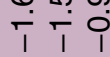

\&

సิ ले

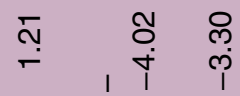

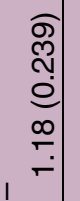

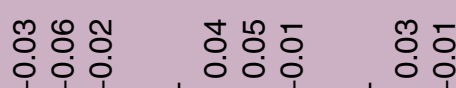

$\begin{array}{llll}8 & 0 & 0 & 0 \\ 0 & 1 & 0 & 1\end{array}$

$\begin{array}{lll}\delta & & \overline{0} \\ 0 & 1 & \\ 0 & 0\end{array}$

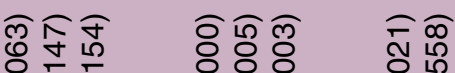

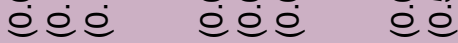

œ ? भ ?

रू

ल

†ั ,

ง

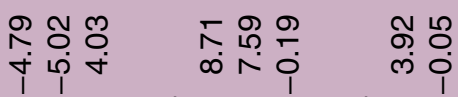

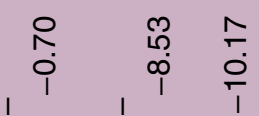

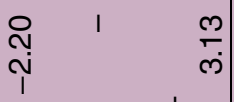

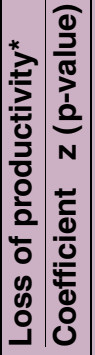

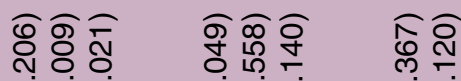

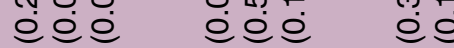

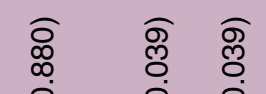

$\begin{array}{ll}\text { o } & \overline{8} \\ 0 & 0 \\ 0 & 0\end{array}$

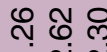

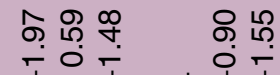

¿

ลำ

ริ

กิ.

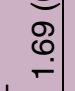

I- ก N

1 1 ।

1 I

ํ.

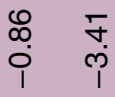

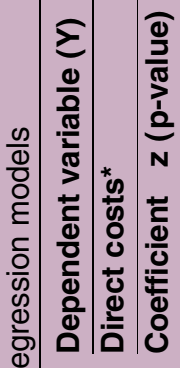

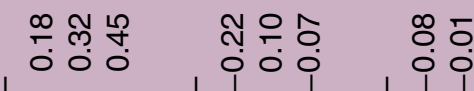

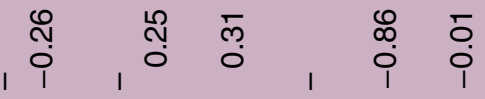
๕ัㅇํㅇำ iอ门

क्ञ

ᄉ

อंอ

สิฐ

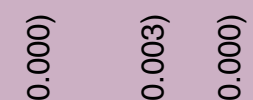

\begin{tabular}{ll} 
तิ \\
พิ \\
\multirow{2}{*}{}
\end{tabular}

N $\infty$

象

พ

$\infty$

อ 0

อ 0

ก

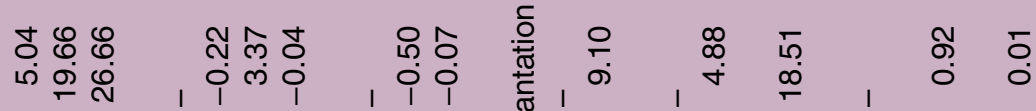

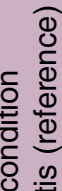

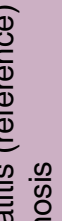

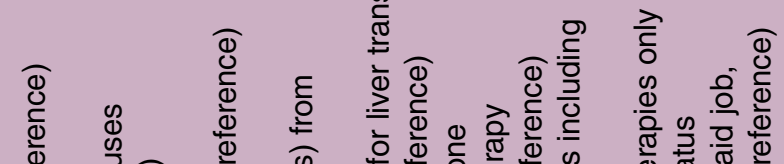

क्षे

\begin{tabular}{l}
5 \\
0 \\
0 \\
0 \\
0 \\
0 \\
0 \\
\hline
\end{tabular} 


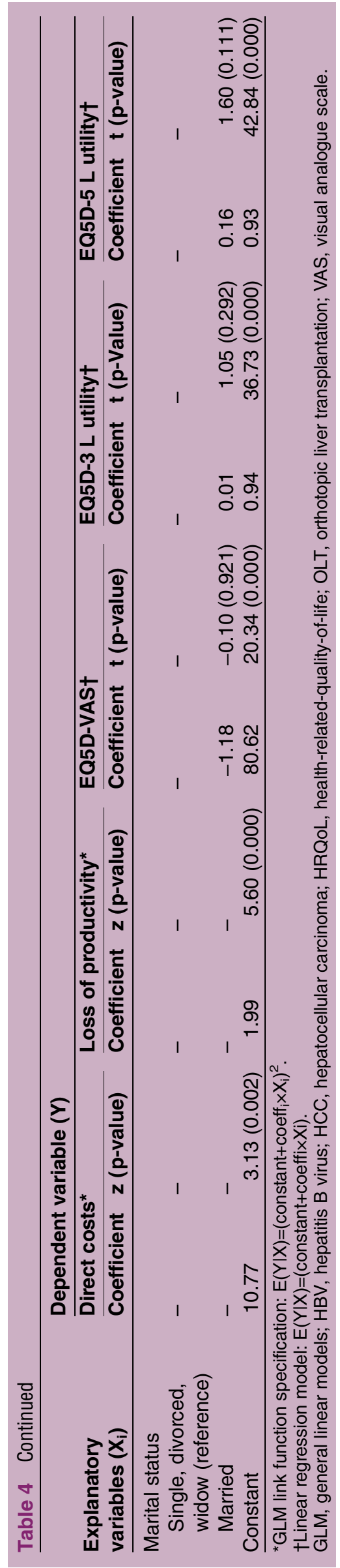

As regards productivity loss, almost half of the patients declared reduced ability to work or to do other everyday activities in the previous 6-month period, and 20 even lost their job due to their hepatic disease. Absenteeism, similarly to direct costs, increased from patients with hepatitis (with an average of around 1 day/patientmonth of absenteeism) to patients with OLT, with up to almost 4 days/patient-month of absenteeism. However, a different trend was found between the aetiological subgroups: mean days lost were lowest among patients with HBV infection, higher among those with HCV infection and followed by those with other aetiologies.

As regards HRQoL, the adjusted score estimates of the VAS and utility indexes presented a sort of $U$ shape between the different disease conditions, corresponding to relatively higher mean levels of HRQoL among hepatitis and patients with OLT, and lower levels among cirrhosis and patients with HCC, using all the scores VAS, 3L utility and 5L utility. Generally, patients with HCV reported the worst mean levels of HRQoL, followed by patients with other aetiologies and then by patients with HBV infection. Several studies have been conducted in the past years to assess HRQoL in the different liver diseases and sequelae. However, most of the studies focused on the diseases caused by HCV infection: the authors of a systematic review published in 2008 on the health-state utilities in liver disease ${ }^{29}$ were able to estimate pooled mean values only for hepatitis $\mathrm{C}$ disease conditions, because all other liver conditions did not have enough estimates to conduct a meta-analyses. From this review, the estimates obtained with the EQ-5D, which was found by the authors as the most frequently used HRQoL tool, were 0.75 for moderate hepatitis $\mathrm{C}$ and compensated cirrhosis, 0.67 for decompensated cirrhosis and 0.71 for OLT. These estimates are lower than ours $(0.90,0.82,0.80$ and 0.83 in chronic hepatitis, cirrhosis, HCC and patients with OLT, respectively), which can depend on a number of reasons, for example, the different methods and different study samples adopted in the different studies, and the different levels of the social tariffs used to obtain utilities: as regards the Italian social tariffs used to obtain utilities in the present study, we found them systematically higher than the widely used UK tariffs. ${ }^{27}$ However, the "U shape" trend of HRQoL levels found in our estimates is confirmed in a systematic review ${ }^{29}$ and in original research that was published in 2008, ${ }^{8}$ reporting the results of utilities estimated from a sample of patients in Singapore with HBV infection diseases at different stages. The authors of this study have specified that while HBV-infected patients reported a not very compromised HRQoL at early stages, from previous literature they found that among hepatitis $\mathrm{C}$ patients, HRQoL is compromised even in those without advanced liver disease. In our study, the lowest mean levels of VAS and utilities were estimated in patients with HCV infection at every disease stage, compared with HBV-infected patients. Also, a direct comparison is available with patients affected from other 

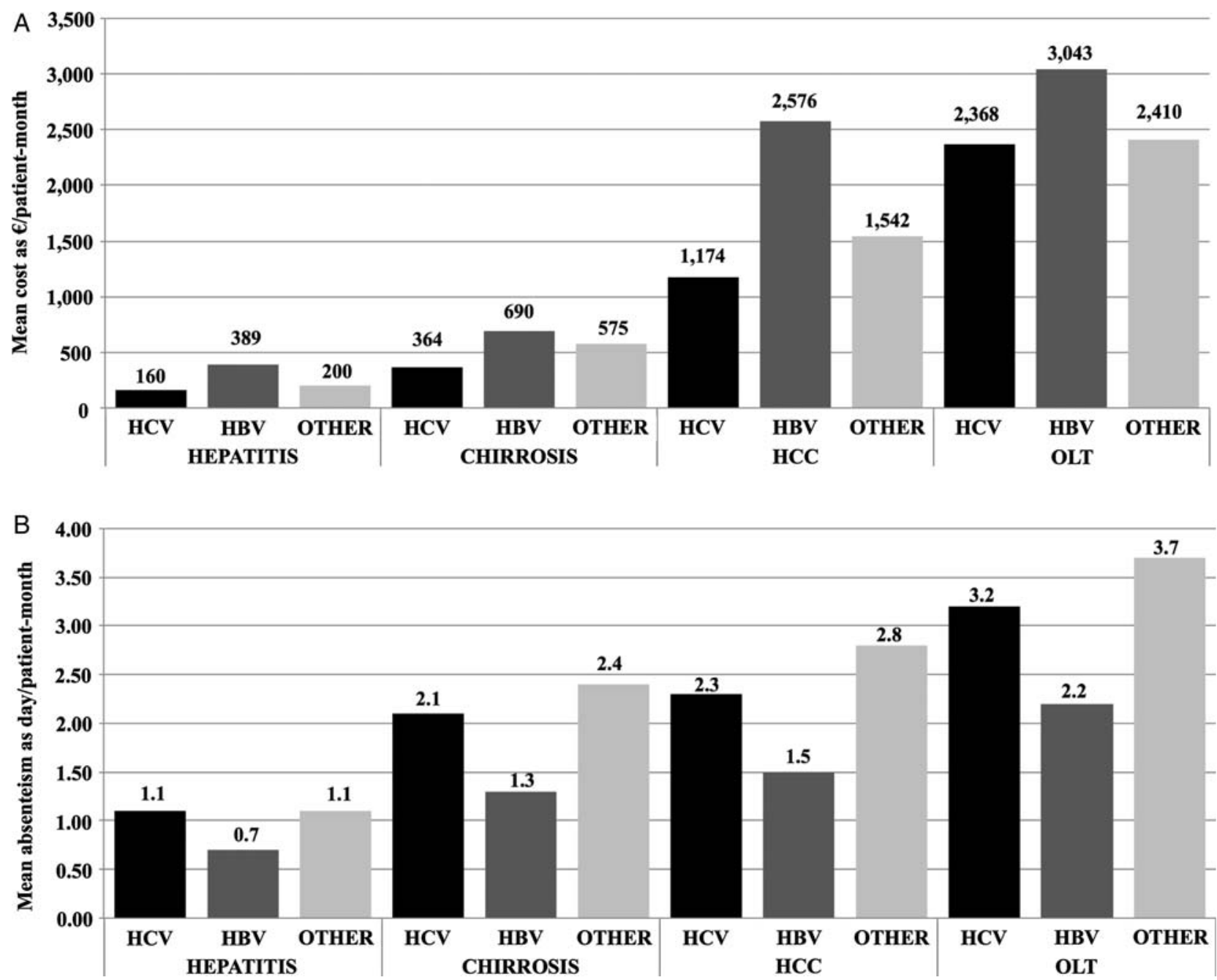

Figure 1 Direct costs as mean €/patient-month (A), absenteeism as mean days/patient-month (B), mean EQ-5D VAS (visual analogue scale) (C), mean EQ-5D-3L utility (D) and mean EQ-5D-5L utility (E) estimated for each disease condition and aetiology, and adjusted for possible confounders. For each parameter, the bars show the trends grouped to distinguish between hepatitis, cirrhosis, hepatocellular carcinoma (HCC) and orthotopic liver transplantation (OLT) patients (groups of three bars per condition), and aetiologies (black bars for hepatitis $\mathrm{C}$ virus (HCV) infection, dark grey bars for hepatitis B virus (HBV) infection, light grey bars for other causes).

causes. Hence, while in their recent review on HRQoL in people with advanced CLDs ${ }^{30}$ the authors underline the controversial results provided by the different studies likely attributable to the different methods applied, with the COME study, we are able to make direct comparisons of HRQoL between the different disease stages and the different aetiological factors.

A limitation that could be ascribed to this study is that it was conducted in two gastroenterology tertiary centres, where it could be expected that only more severe patients are treated and were involved in the COME study. The centres involved in the study manage CLDs starting from early stages until the very complex post-transplant stages. Therefore, we do not believe that the study sample involved in this study could have generated biased estimates.

A second limitation is that while we report the results on the 'other causes' category of patients, we do not specify any details according to these patients' specific clinical condition: the low number of patients belonging to each subcategory would not be suitable to obtain reliable and useful estimates. In any case, our estimates remain useful to identify the burden of CLDs in this category of participants, who show high levels of costs and compromised HRQoL, and could stimulate questions and hypotheses to investigate on future ad hoc research.

A third potential limitation of this study is that it was conducted only in Italy, thus potentially limiting the external validity of our results, as different cost items and/or different unit costs are generally applicable in the different healthcare systems, with possibly different results. Owing to these differences in the healthcare systems, carrying out accurate and precise international COI studies in the different healthcare areas is generally very difficult. Hence, a compromise must be reached to conduct a methodologically correct and informative 

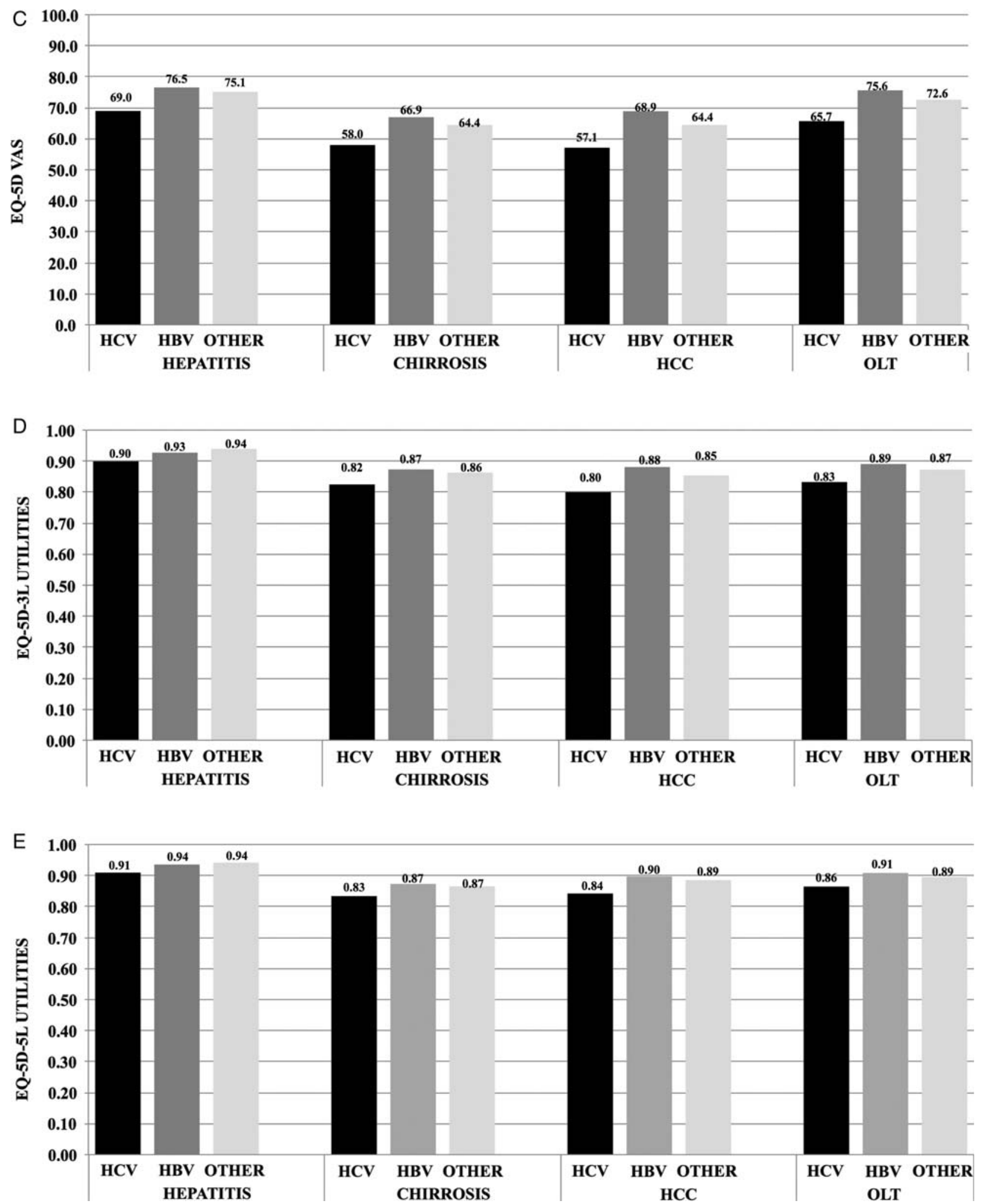

Figure 1 Continued

scientific work. A study has recently been conducted in five European countries to assess costs and HRQoL from the patients' perspective. ${ }^{10}$ However, some drawbacks were admitted to in that study: because a consumer panel was used to recruit the patients, it was not possible to confirm the target diagnosis among the participants; furthermore, the costs attributable to the condition were not estimated directly, but derived from a comparison of costs with a sample of controls. Finally, the study focused only on HCV infection. In contrast, by conducting our 
study through gastroenterology hospitals, we involved patients with a confirmed diagnosis of the different CLDs. Information necessary to conduct the analyses was supervised or reported by the doctor and data on direct cost and productivity loss referred specifically to the hepatic condition. Finally, we adopted the perspective of the healthcare system and the perspective of patients and their families. Therefore, while on one hand the study by Vietri $e t a l^{10}$ was conducted in several European countries, on the other, our Italian study can be considered more complete as regards the greater number of hepatic conditions considered and the more complete estimates obtained. Hence, until an international COI study able to provide complete and precise estimates on the burden of CLDs is available, the results of the present study can be considered the most complete available and generally applicable also in other countries with a similar gross domestic product. Furthermore, it can give some insights on the approach and methods that can be applied or adapted in other healthcare systems for the conducting of similar studies in other countries.

CLDs affect participants for many years, making observation of the natural history of these conditions very difficult, or even impossible. Despite this being a retrospective and prevalence-based study, that is, not able per se to prospectively follow patients from the onset of their conditions, the involvement of a relatively large and heterogeneous study sample covering all main liver conditions from different aetiologies allowed us to build a picture of this complex phenomenon from many different aspects. This study provides useful information to plan a strategy for the healthcare system in order to decide the best investment. These results are even more important in the light of new antivirals, as they provide information that can be used to conduct accurate pharmacoeconomic analyses aimed at understanding the cost-effectiveness of the new interventions.

A further novelty of this study is that it also provides utility values obtained using the Italian specific social tariffs, the standard and widely used EQ-5D-3L, ${ }^{8}{ }^{29}$ and the recently introduced EQ-5D-5L descriptive system which will probably be used frequently in future research, in pharmacoeconomic evaluations and in decision-making. ${ }^{31-33}$ Notice that the utility estimates shown in this paper are different from those presented in the paper by Scalone et $a l,{ }^{26}$ because at the time of the publication of that paper, the Italian social tariffs ${ }^{27}$ were not yet available. Hence, the utility indexes reported in this paper are to be considered more accurate than those published previously.

To conclude, CLDs compromise patients' well-being and generate high costs for the healthcare system and the society as a whole. Costs increase and HRQoL worsens with disease progression. The use of effective treatments in the early stages of liver diseases appears necessary when aiming at reducing worsening of patient health, and direct and indirect costs. The non-availability of effective aetiological treatments for the most frequent liver dysfunctions (HBV and HCV) has limited the effectiveness of interventions aimed at preventing the evolution of such diseases. The availability of new antiviral therapies for both these virusrelated liver diseases is expected to change the medical approach towards patients and their treatment. A proper knowledge of the global socioeconomic burden of CLDs is paramount to plan adequate screening and treatment policies aimed at maximisation cost-effectiveness of interventions.

\section{Author affiliations}

${ }^{1}$ Centro di Studio e Ricerca sulla Sanità Pubblica (CESP), Università degli Studi di Milano Bicocca, Monza, Italy

${ }^{2}$ Fondazione CHARTA, Milano, Italy

${ }^{3}$ Gastroenterologia, Epatologia e Trapiantologia Ospedale Papa Giovanni XXIII, Bergamo, Italy

${ }^{4}$ Organizzazione di pazienti-EpaC ONLUS

${ }^{5}$ Unità di Malattie Infettive Tropicali e Epatologia Dipartimento di Scienze Clinico-Chirurgiche Diagnostiche e Pediatriche, Università degli Studi di Pavia, Italy

${ }^{6}$ Dipartimento Malattie Infettive Fondazione IRCCS Policlinico San Matteo, Pavia, Italy

${ }^{7}$ Istituto di Management-Management e Innovation (MAIN) Scuola Superiore Sant'Anna, Pisa, Italy

${ }^{8}$ Gastroenterologia. Dipartimento di Medicina Clinica e Chirurgia. Scuola di Medicina, Università degli Studi di Napoli Federico II, Italy

Acknowledgements The authors thank all the patients who agreed to participate in the present study. The authors also thank Anna Baldan and Giulia Magini, who cooperated in drafting the questionnaire, and to Giovanna Gaffuri for her contribution to data collection. The authors especially thank Paolo Cozzolino, who gave technical support in preparing this manuscript, and thank Paolo Angelo Cortesi, who contributed to the literature research and provided technical comments during data analyses and manuscript preparation.

Contributors Each of the listed authors has given substantial contribution to conception and design, or acquisition of data, or analysis and interpretation of data, drafting the article or revising it critically. In particular LS, SF, RC, IG and LGM designed the study. SF, LP, MGL, LG and ADP collected the data, LS, RC and FF analysed the data. LS, SF, RC, RB, IG, GC and LGM interpreted the results and wrote the paper. All the authors have accepted the final version of the paper.

Funding Funds were provided by EpaC Onlus Patients' Associations and CHARTA Foundation. An educational contribution was also provided by Schering Plough, Janssen Cilag and Gilead Sciences. The funding agreement ensured the authors' independence in designing the study, interpreting the data, and writing and publishing the paper.

Competing interests SF is on the speaker's bureau of: Gilead Sciences, BMS, MSD, Janssen Cilag, Novartis, Bayer, AbbVie, Roche, Grifols, Kedrion and Biotest. LGM received grants from MSD and Janssen Cilag. He consults for and is on the speakers' bureau of Bayer. RB has acted as an advisory and/or speaker receiving fees from AbbVie, Boehringer Ingelheim, Gilead Sciences, Janssen Cilag, MSD and Roche.

Ethics approval Ethical Committees of the participant hospital centres.

Provenance and peer review Not commissioned; externally peer reviewed.

Data sharing statement No additional data are available.

Open Access This is an Open Access article distributed in accordance with the Creative Commons Attribution Non Commercial (CC BY-NC 4.0) license, which permits others to distribute, remix, adapt, build upon this work 
non-commercially, and license their derivative works on different terms, provided the original work is properly cited and the use is non-commercial. See: http://creativecommons.org/licenses/by-nc/4.0/

\section{REFERENCES}

1. European Centre for Disease Prevention and Control. Hepatitis $B$ and Cin the EU neighborhood: prevalence, burden of disease and screening policies. 2010.

2. Sorrell MF, Belongia EA, Costa J, et al. National Institutes of Health Consensus Development Conference Statement: management of hepatitis B. Ann Intern Med 2009;150:104-10.

3. Perz JF, Armstrong GL, Farrington LA, et al. The contributions of hepatitis $B$ virus and hepatitis $C$ virus infections to cirrhosis and primary liver cancer worldwide. J Hepatol 2006;45:529-38.

4. NIH Consensus Statement on Management of hepatitis C: 2002. NIH Consens State Sci Statements 2002;19:1-46.

5. Fattovich G, Stroffolini T, Zagni I, et al. Hepatocellular carcinoma in cirrhosis: incidence and risk factors. Gastroenterology 2004;127: S35-50.

6. Thein $\mathrm{HH}, \mathrm{Yi} \mathrm{Q}$, Dore GJ, et al. Estimation of stage-specific fibrosis progression rates in chronic hepatitis $\mathrm{C}$ virus infection: a metaanalysis and meta-regression. Hepatology 2008;48:418-31.

7. Hatzakis A, Wait S, Bruix J, et al. The state of hepatitis $B$ and $C$ in Europe: report from the hepatitis B and $\mathrm{C}$ summit conference. J Viral Hepat 2011;18(Suppl 1):1-16.

8. Ong SC, Mak B, Aung MO, et al. Health-related quality of life in chronic hepatitis B patients. Hepatology 2008;47:1108-17.

9. Hsu PC, Federico CA, Krajden M, et al. Health utilities and psychometric quality of life in patients with early- and late-stage hepatitis C virus infection. J Gastroenterol Hepatol 2012;27:149-57.

10. Vietri J, Prajapati G, El Khoury AC. The burden of hepatitis C in Europe from the patients' perspective: a survey in 5 countries. BMC Gastroenterology 2013;13:16.

11. Federico CA, Hsu PC, Krajden M, et al. Patient time costs and out-of-pocket costs in hepatitis C. Liver Int 2012;32:815-25.

12. Khoury AC, Klimack WK, Wallace C, et al. Economic burden of hepatitis C-associated diseases in the United States. J Viral Hepat 2012;19:153-60.

13. Neff GW, Duncan CW, Schiff ER. The current economic burden of cirrhosis. Gastroenterol Hepatol (N Y) 2011;7:661-71.

14. McCombs JS, Yuan Y, Shin J, et al. Economic burden associated with patients diagnosed with hepatitis C. Clin Ther 2011;33:1268-80.

15. Su J, Brook RA, Kleinman NL, et al. The impact of hepatitis C virus infection on work absence, productivity, and healthcare benefit costs. Hepatology 2010;52:436-42.

16. Yang BM, Kim DJ, Byun KS, et al. The societal burden of HBV-related disease: South Korea. Dig Dis Sci 2010;55:784-93.
17. Ong SC, Lim SG, Li SC. How big is the financial burden of hepatitis $B$ to society? A cost-of-illness study of hepatitis B infection in Singapore. J Viral Hepat 2009;16:53-63.

18. Gutteling JJ, de Man RA, Busschbach JJ, et al. Overview of research on health-related quality of life in patients with chronic liver disease. Neth J Med 2007;65:227-34.

19. Grant WC, Jhaveri RR, McHutchison JG, et al. Trends in health care resource use for hepatitis $C$ virus infection in the United States. Hepatology 2005;42:1406-13.

20. Olivieri I, de Portu S, Salvarani C, et al. The psoriatic arthritis cost evaluation study: a cost-of-illness study on tumour necrosis factor inhibitors in psoriatic arthritis patients with inadequate response to conventional therapy. Rheumatology 2008;47:1664-70.

21. World Medical Association (WMA) Declaration of Helsinki. Adopted by the 18th World Medical Assembly, Helsinki, Finland, June 1964, amended by the 29th World Medical Assembly, Tokyo, Japan, October 1975, the 35th World Medical Assembly, Venice, Italy, October 1983 and the 41st World Medical Assembly, Hong Kong, September 1989.

22. Prezzi dei farmaci. http://farmaco.agenziafarmaco.it/index.php, visited in January 2011.

23. Tariffari regionali delle prestazioni di assistenza ospedaliera" provided under the hospitalization and day-hospital. http://www. agenas.it/monitoraggio_costi.html, visited in July 2011.

24. Prestazioni specialistiche ambulatoriali-confronto tra le tariffe nazionali ex DM 1996 e le tariffe regionali massime relative all'anno 2008. http://www.agenas.it/monitoraggio_costi_tariffe/monitoraggio_ costi_tariffe_prestazioni.htm visited in January 2011

25. Rabin R, de Charro F. EQ-5D: a measure of health status from the EuroQoL Group. Ann Med 2001;33:337-43.

26. Scalone L, Ciampichini R, Fagiuoli S, et al. Comparing the performance of the standard EQ-5D 3L with the new version EQ-5D $5 \mathrm{~L}$ in patients with chronic liver diseases. Qual Life Res 2013;22:1707-16.

27. Scalone L, Cortesi PA, Ciampichini R. Italian population-based values of EQ-5D health states. Value Health 2013;16:814-22.

28. van Hout $B$, Janssen MF, Feng $Y S$, et al. Value sets for the EQ-5D-5L: a mapping approach. Value Health 2012;15:708-15.

29. McLernon DJ, Dillon J, Donnan PT. Health-state utilities in liver disease: a systematic review. Med Decis Making 2008;28:582-92.

30. Orr JG, Homer T, Ternent L, et al. Health related quality of life in people with advanced chronic liver disease. $J$ Hepatol 2014;61:1158-65.

31. Krabbe PF, Devlin NJ, Stolk EA, et al. Multinational evidence of the applicability and robustness of discrete choice modeling for deriving EQ-5D-5 L health-state values. Med Care 2014;52:935-43.

32. Oppe M, Devlin NJ, van Hout B, et al. A program of methodological research to arrive at the new international $E Q-5 D-5 L$ valuation protocol. Value Health 2014;17:445-53.

33. Devlin NJ, Krabbe PF. The development of new research methods for the valuation of EQ-5D-5L. Eur J Health Econ 2013;14(Suppl 1):S1-3. 